\title{
The implications of organizational environment questionnaire for the assessment of occupational injury among medical workers
}

\author{
Xuan $\mathrm{HU}^{1}$, Shu $\mathrm{CAI}^{2}$, Hong $\mathrm{LIN}^{3}$, Jin-Dong XU ${ }^{4}$, Jin-Guo ZHAI ${ }^{5}$, Wen-Zhi CAI ${ }^{6 *}$
}

\begin{abstract}
Organization environment has significant influence on occupational health among medical workers. In current study, based on previous established questionnaires, we constructed a new questionnaire to evaluate the effects of organizational environment on occupational injury of medical workers. In a large-scale of investigation, a total of 2350 medical workers from 54 hospitals in Zhuhai city, China, were surveyed by the new questionnaire, and 2111 (91.2\%) questionnaires were used for final analysis. The average score of organization environment of medical workers questionnaire was $91.33 \pm 21.95$. We found that as compared with the average levels, medical workers have higher incidences of behavioral symptoms such as depressed mood, obsessional thinking, and anxiety and so on. We also demonstrated the questionnaire scores correlated with the incidence of occupational disease, occupational injuries and the scores of SCL-90, implying its potential application to objectively measure the conditions of medial safety of medical workers in China. In all, we concluded that organizational environment has important implications on the assessment of occupational health of medical workers.
\end{abstract}

Keywords: medical workers; occupational health; occupational injury; organization environment.

Practical Application: The new version of organizational environment questionnaire and confirm its reliability and validity in a large-scale of observational investigation.

\section{Introduction}

AS the evolution of social environment and increases of occupational risk factors, medical workers are faced with multiple occupational injuries. According to the previous data of United States Department of Labor in 2005, nursing industry has again ranked top one on the incidences of occupational injuries in US (Gershon et al., 2007). Additionally, investigations have shown that medical workers are susceptible to hepatitis viruses' infections and needle stick injuries (Centers for Disease Control and Prevention, 2007; Trinkoff et al., 2007). Trinkoff et al. reported that the prevalence of musculoskeletal diseases was dramatically elevated in nurses, which implies the necessity to deal with musculoskeletal diseases in nurses (Trinkoff et al., 2006). Stone et al.reported that over $75 \%$ of nurses got sick due to the work overload (Stone et al., 2007). However, this situation is only one part of the story. In fact, mental health is the major field where medical workers were affected. Studies have reported high prevalence of depression, anxiety, chronic stress, insomnia, suicide in medical workers (Ruitenburg et al., 2012). A survey from Netherland revealed that the occurrence of anxiety and depression symptoms in doctors were $24 \%$ and $29 \%$, respectively (Schwenk et al., 2008). Another investigation from South Korea showed that $44 \%$ of nurses had depression

among the 441 registered nurses observed (Yoon \& Kim, 2013). In China, the occupational health of medical workers has drawn attentions from different social levels. Based on the survey made by the Minister of Health of the People's Republic of China, in 2010 , it was estimated that $25 \%$ of 4032 doctors had cardiovascular diseases and over $50 \%$ of them had hypertension. In addition, the situation of mental health in medical workers in China can also not be optimistic and the rate of suicides of doctors was dramatically increased in recent years. Moreover, food have significant effect on the health of medical workers (Yildiz et al., 2021; Hossen et al., 2021; Ein Ali Afjeh et al., 2020).

Organizational environment plays an important role in the management of occupational injuries in medical workers (Zohar, 1980). A hypothesis have been proposed that any injuries or accidents can be attributed to the dysregulation of management and organizational factors within the group. In the past decades, some questionnaires and scales have been created to evaluate the influence of organizational environment on work-related injury, job satisfaction, and occupational fatigue and so on (Vogus \& Sutcliffe, 2007; Gershon et al., 2007; Gimeno et al., 2005). In 2000, hospital safety climate scale (HSCS) was developed by Robyn

${ }^{1}$ School of Nursing, Guangzhou Medical University, Guangzhou, Guangdong Province, China

${ }^{2}$ School of Nursing, Guangdong Pharmaceutical University, Guangzhou, Guangdong Province, China

${ }^{3}$ Phoenix International Medical Center, The Fifth Affiliated Hospital of Sun Yat-Sen University, Zhuhai, Guangdong Province, China

${ }^{4}$ Department of Anesthesiology, Guangdong Cardiovascular Institute, Guangdong General Hospital, Guangdong Academy of Medical Science, Guangzhou, Guangdong

Province, China

${ }^{5}$ School of Nursing, Southern Medical University, Guangzhou, Guangdong Province, China

${ }^{6}$ President's Office, Shenzhen Hospital of Southern Medical University, Shenzhen, Guangdong Province, China

*Corresponding author: caiwenzhi2018@126.com 
Gershon, which has become one of the most universally used instruments for the assessment of hospital safety (Gershon et al., 2007). The Chinese version of HSCS has been created and contained five dimensions composed of 21 items. The safety climate questionnaire (SCQ) is another scale generally used for the evaluation of safety climate in hospital and has been adapted for the measure the influences of organizational management on the medical injury in China (Gimeno et al., 2005).

To decipher the role of organizational environment on occupational health of medical workers, we revised the Chinese version of organizational environment questionnaire and further examined its reliability and validity for clinical application. Then we made a survey containing 2350 medical workers from 54 hospitals in Zhuhai, China, and further studied the relationships between medical injury and organizational environment. Finally, based on our data, we recommended that organizational environment questionnaire can be used for the assessment of occupational health of medical workers in China.

\section{Methods and participates}

\subsection{Subjects and observational protocol}

A multi-stage stratified sampling was performed in 3 administrative regions and 5 economic function areas of Zhuhai city, China. There are totally 16637 medical workers randomly sampled in 54 hospitals from above geographical regions and finally 2350 workers responded and 2111 of the finished questionnaires are selected for following analysis.

This study was investigated with the electronic questionnaires and all of the subjects finished the Infomart consent forms before accepting to reply the electronic questionnaire Email.

\subsection{Questionnaire establishment}

According to the published literature and current extant research consents, the new version of the questionnaire was created and contains four parts: general information, mental health, occupational injury, and organizational environment. The general information included the basic demographic data of the subjects surveyed. The mental health was evaluated by the Symptom Checklist 90 (SCL-90). The occupational injury was assessed based on the assessed items published in previous studies. For the construction of organizational environment questionnaire, firstly, an initial version of the new questionnaire was created based on the past literature by searching following keywords: organizational environment administration, hospital staff, occupational injury, occupational health, and occupational exposure and previous established scales. After the initial review of literature, the first version of questionnaire was discussed by a group of experts who had ample experience on epidemiological investigations. Consensus was made to confirm the contents of the final questionnaire. The reliability of the questionnaire was assessed using Cronbach's a (Supplemental Table 1). The higher the $\alpha$ is, the stronger the reliability of questionnaire will be. We also used corrected item-total correction (CITC) to further check the reliability of the items (Supplemental Table 2, 3, 4, 5 and 6). An item can be selected only under two conditions: CITC is less than 0.5 and the global reliability can be improved by deleted the item from the questionnaire.The final items contained in the questionnaire was scored based on a ranking score criteria for the dimensions of organization environment questionnaire, which was shown in Supplemental Table 7.

\subsection{Statistical analysis}

The data was shown as Mean \pm SD. The statistical analysis was performed using SPSS (version 21.0) and AMOS (version 21.0). Cronbach's a was calculated to assess internal consistency of the reliability. Pearson correlation analysis was used for correlation analysis of different dimensions within the questionnaire and relationships between organizational environment, occupational injury, occupational disease, and SLC-90. KMO test and Bartlett test were used to evaluate whether the data was suitable for factorial analysis and principle component analysis. Unpaired $\mathrm{t}$ test was used for the comparison of means of two groups and ANOVA was used for the comparisons of multiple groups. $\mathrm{X}^{2}$ test was used for the comparison of disease incidence and positive screening rate. A P value less than 0.05 was considered to be statistically different.

\section{Results}

\subsection{The reliability and validity of revised organizational environment questionnaire}

The Pearson correlation analysis of 32 items scores and total score of questionnaire revealed that the Pearson coefficients analyzed ranged from 0.489 to 0.747 (Table 1). All Pearson coefficients of the items were over 0.4 and thus no item was considered redundant. We found that the global Cronbach's a coefficient of revised organizational environment questionnaire is 0.962 , and the Cronbach's a coefficients of five dimensions varied from 0.828 to 0.945 (Table 2).

The KMO test and Bartlett test demonstrated that the data can be analyzed by factorial analysis. The results of factorial analysis showed that every item had relative contribution to the global variance (Supplemental Table 8). By combining the Scree plot (Figure 1) with our theoretical framework, we extracted five common factors to establish the new questionnaires and demonstrated that all of them showed higher workload. On

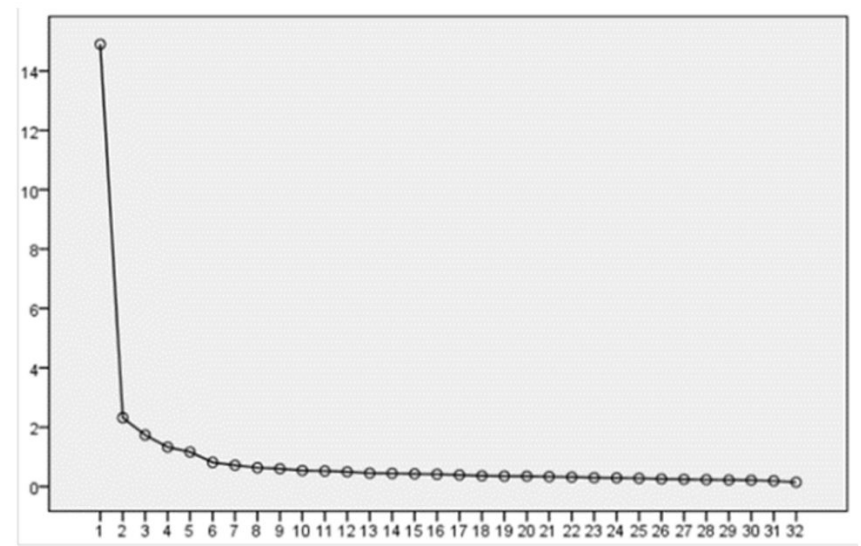

Figure 1. The Scree plot of factorial analysis. 
the basis of questionnaires, we used AMOS 21.0 for testified factorial analysis and created the second order model to adapt the questionnaire. The results showed that the primary model had good fitness and the adjusted model satisfied the requirements of good models (Table 3).

\subsection{The correlation between organizational environment and occupational injury and occupational disease}

In total, 2111 questionnaires were collected and analyzed. Five hundred and two of them are male, and 1609 of them are female. The average age of the respondents was $33.04 \pm 8.74$. The detailed demographic data and characteristics of the respondents has been shown in Table 4. The incidences of occupational injuries and occupational diseases in our group were shown in Table 5 and Table 6. By using Pearson correlation analysis, we revealed that scores of organizational environment questionnaire significantly different occupational injury status (Table 7) and occupational diseases (Table 8).

\subsection{The correlations between organizational environment and mental health}

For the assessment of metal health, we found that as compared with the average levels, medical workers have higher incidence of behavioral symptoms, including depressed mood, obsessional thinking, and anxiety and so on (Table 9). By using
Pearson correlation analysis, we found that scores of organization environment questionnaire significantly correlated with scores of SCL-90 (Table 10).

\subsection{The correlation between mental health and occupational injury occupational injuries}

Pearson correlation analysis showed that scores of SCL-90 significantly correlated with occupational injury (Table 11).

\section{Discussion}

In the industrial setting, employee understandings regarding their organization's commitment to safety (i.e., safety climate) are demonstrated to be essential correlates to both the execution and maintenance of safe work practices and to the incidence of work related injuries. However, safety climate evaluation specific to the medical setting remains to be a field required to be explored, especially for China. Previously, some questionnaires and scales have been developed for the assessment of safety climate in specific medical situations, such as HSCS and SCQ. In China, the Chinese version of HSCS had been created and the research on the issue of medical safety for medical workers also began to arise. As one of the part of safety climate, organizational environment plays an important role in maintenance of medical safety in medical workers.

Table 1. The Pearson correlation coefficient matrix between scores of each item and total score.

\begin{tabular}{|c|c|c|c|c|c|c|c|}
\hline Item & Coefficient & Item & Coefficient & Item & Coefficient & Item & Coefficient \\
\hline BA1 & $0.502^{* *}$ & BB4 & $0.730^{* *}$ & $\mathrm{BD} 2$ & $0.633^{* *}$ & BE3 & $0.720^{\star *}$ \\
\hline BA2 & $0.522^{\star *}$ & BB5 & $0.707^{\star *}$ & BD3 & $0.689^{* *}$ & BE4 & $0.724^{* *}$ \\
\hline BA3 & $0.489^{* *}$ & BC1 & $0.504^{* *}$ & BD4 & $0.512^{* *}$ & BE5 & $0.638^{\star *}$ \\
\hline BA4 & $0.525^{\star *}$ & BC2 & $0.521^{\star *}$ & BD5 & $0.591^{\star *}$ & BE6 & $0.685^{\star *}$ \\
\hline BA5 & $0.600^{* *}$ & BC3 & $0.592^{\star *}$ & BD6 & $0.673^{\star *}$ & BE7 & $0.731^{\star *}$ \\
\hline BB1 & $0.630^{* *}$ & BC4 & $0.667^{\star *}$ & BD7 & $0.671^{* *}$ & BE8 & $0.718^{\star *}$ \\
\hline BB2 & $0.616^{\star *}$ & BC5 & $0.569^{\star *}$ & BE1 & $0.694^{\star *}$ & BE9 & $0.732^{\star *}$ \\
\hline BB3 & $0.657^{\star *}$ & BD1 & $0.577^{\star \star}$ & BE2 & $0.662^{\star *}$ & BE10 & $0.747^{\star *}$ \\
\hline
\end{tabular}

Table 2. The correlation coefficient matrix of dimensions of organization environment questionnaire.

\begin{tabular}{|c|c|c|c|c|c|}
\hline Item & Manpower resource & Work environment & Facilities & Organizational culture & Management support \\
\hline Manpower resource & $0.828^{\#}$ & & & & \\
\hline Work environment & $0.605^{\star *}$ & $0.912^{\#}$ & & & \\
\hline Facilities & $0.525^{\star \star}$ & $0.530^{\star *}$ & $0.845^{\#}$ & & \\
\hline Organizational culture & $0.484^{* *}$ & $0.540^{* *}$ & $0.639^{* *}$ & $0.915^{\#}$ & \\
\hline Management support & $0.583^{\star *}$ & $0.737^{\star *}$ & $0.626^{\star \star}$ & $0.673^{\star *}$ & $0.945^{\#}$ \\
\hline
\end{tabular}

${ }^{* *} \mathrm{P}<0.01$; ${ }^{*}$ Represents the Cronbach's a coefficient, others are Pearson coefficients.

Table 3. Fit indices of the CFA structural models.

\begin{tabular}{|c|c|c|c|c|c|c|c|c|}
\hline Fitness index & RFI & GFI & AGFI & CFI & NFI & TLI & SRMR & RMSEA \\
\hline Refer criterion & $>0.9$ & $>0.9$ & $>0.9$ & $>0.9$ & $>0.9$ & $>0.9$ & $<0.05$ & $<0.08$ \\
\hline Before adjustment & 0.886 & 0.847 & 0.823 & 0.903 & 0.895 & 0.958 & 0.044 & 0.068 \\
\hline After adjustment & 0.939 & 0.905 & 0.920 & 0.954 & 0.945 & 0.945 & 0.039 & 0.049 \\
\hline
\end{tabular}

RFI: Relative Fit Index; GFI: Goodness-of -Fit Index; AGFI: Adjusted GFI; CFI: Comparative Fit Index; NFI: Normed Fit Index; TLI: Turk-Lewis Index; SRMR: Standardized Root Mean Square Residual; RMSEA: Root Mean Square Error of Approximation. 
Table 4. The demographic data of the subjects surveyed.

\begin{tabular}{|c|c|}
\hline Group & Percentage \\
\hline \multicolumn{2}{|l|}{ Age(y) } \\
\hline$\leq 25$ & $21 \%$ \\
\hline $26-35$ & $45 \%$ \\
\hline $36-45$ & $24 \%$ \\
\hline 46 & $10 \%$ \\
\hline \multicolumn{2}{|l|}{ Education } \\
\hline Junior college & $31 \%$ \\
\hline Undergraduate & $49 \%$ \\
\hline Master or $\mathrm{PhD}$ & $7 \%$ \\
\hline \multicolumn{2}{|l|}{ Length of service } \\
\hline$\leq 5$ & $38 \%$ \\
\hline \multicolumn{2}{|l|}{ The level of hospital } \\
\hline Grade 1 & $35 \%$ \\
\hline Grade 2 & $27 \%$ \\
\hline Grade 3 & $38 \%$ \\
\hline \multicolumn{2}{|l|}{ Department distribution } \\
\hline Surgery & $15 \%$ \\
\hline Internal medicine & $51 \%$ \\
\hline Assisted medical technician & $16 \%$ \\
\hline Administrative management & $12 \%$ \\
\hline Others & $6 \%$ \\
\hline$\leq 2000$ & $9 \%$ \\
\hline $2001-4000$ & $41 \%$ \\
\hline $4100-6000$ & $23 \%$ \\
\hline $6001-8000$ & $15 \%$ \\
\hline$>8000$ & $12 \%$ \\
\hline
\end{tabular}

Table 5. The incidence rate of occupational injury.

\begin{tabular}{|c|c|c|c|c|c|}
\hline Order & Type & Incidence (\%) & Order & Type & Incidence (\%) \\
\hline 1 & Oral ulcer & 62.0 & 11 & Fall damage & 21.8 \\
\hline 2 & Direct impact & 45.7 & 12 & Scratch & 16.7 \\
\hline 3 & Menoxenia & 44.5 & 13 & Leukopenia & 11.1 \\
\hline 4 & Pricking wound & 44.3 & 14 & Strike injury & 5.5 \\
\hline 5 & Allergic dermatitis & 36.8 & 15 & Burn injury & 5.0 \\
\hline 6 & Concis & 36.6 & 16 & Epidemic keratitis & 4.8 \\
\hline 7 & Rash & 36.5 & 17 & frostbite & 4.5 \\
\hline 8 & Chapped skin & 35.2 & 18 & Electrical lesion & 2.1 \\
\hline 9 & Sprain & 32.5 & 19 & Other & 1.2 \\
\hline 10 & Pull & 26.2 & & & \\
\hline
\end{tabular}


Original Article

Hu et al.

Table 6. The incidence rate of occupational diseases.

\begin{tabular}{cccccc}
\hline Order & Occupational disease & Incidence $(\%)$ & order & Occupational disease & Incidence (\%) \\
\hline $\mathbf{1}$ & Cervical/Lumbar spondylosis & 33.5 & 13 & Other & 2.0 \\
$\mathbf{2}$ & Chronic pharyngitis & 20.7 & 14 & Coronary artery disease & 1.9 \\
$\mathbf{3}$ & Neurasthenia & 20.2 & 15 & Pneumoniae & 1.8 \\
$\mathbf{4}$ & Gastric ulcer & 15.5 & 16 & Tuberculosis & 1.6 \\
$\mathbf{5}$ & Chronic rhinitis & 15.3 & 17 & Radiation sickness & 1.1 \\
$\mathbf{6}$ & varicose of lower limb & 13.3 & 18 & Cancer & 1.1 \\
$\mathbf{7}$ & Depression & 6.4 & 19 & Asthma & 0.6 \\
$\mathbf{8}$ & Carpal tunnel syndrome & 4.2 & 20 & Cataracts & 0.6 \\
$\mathbf{9}$ & Auditory nerve impairment & 3.5 & 21 & Bone fracture & 0.0 \\
$\mathbf{1 0}$ & Premature ejaculation & 3.3 & 22 & AIDs & 0.0 \\
$\mathbf{1 1}$ & HAV/HBV/HCV hepatitis & 2.7 & 23 & SARS & \\
$\mathbf{1 2}$ & Primary hypertension & 2.2 & & & \\
\hline
\end{tabular}

Table 7. Comparison of scores of organization environment questionnaire between different occupational injury status.

\begin{tabular}{|c|c|c|c|c|c|c|}
\hline \multirow{2}{*}{ Variant } & \multicolumn{2}{|c|}{ Oral ulcer } & \multirow{2}{*}{$\mathrm{t}$} & \multicolumn{2}{|c|}{ Stick injury } & \multirow{2}{*}{$\mathrm{t}$} \\
\hline & Yes & No & & Yes & No & \\
\hline Organizational environment ${ }^{\#}$ & $89.47 \pm 21.492$ & $94.37 \pm 22.36$ & $4.990^{* *}$ & $87.20 \pm 20.91$ & $94.63 \pm 22.20$ & $7.841^{* *}$ \\
\hline Manpower resource & $14.80 \pm 3.34$ & $15.40 \pm 3.47$ & $3.909^{* *}$ & $14.56 \pm 3.29$ & $15.4 \pm 3.45$ & $5.658^{* *}$ \\
\hline Work environment & $11.83 \pm 4.21$ & $12.78 \pm 4.60$ & $4.743^{* *}$ & $11.52 \pm 4.09$ & $12.72 \pm 4.54$ & $6.383^{* \star}$ \\
\hline Facilities & $16.02 \pm 3.16$ & $16.49 \pm 3.29$ & $3.266^{* *}$ & $15.66 \pm 3.08$ & $16.63 \pm 3.27$ & $6.920^{* *}$ \\
\hline Organizational culture & $22.95 \pm 5.58$ & $23.60 \pm 5.60$ & $2.573^{* *}$ & $22.32 \pm 5.52$ & $23.91 \pm 5.56$ & $6.560^{* *}$ \\
\hline Management Support & $26.85 \pm 8.35$ & $28.85 \pm 8.59$ & $5.261^{* *}$ & $25.97 \pm 8.23$ & $28.92 \pm 8.48$ & $8.033^{* *}$ \\
\hline
\end{tabular}

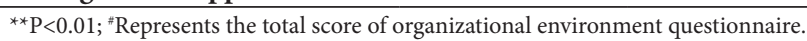

Table 8. Comparison of scores of organization environment questionnaire between different occupational diseases.

\begin{tabular}{|c|c|c|c|c|c|c|c|c|c|}
\hline \multirow{2}{*}{ variant } & \multicolumn{2}{|c|}{ Cervical/Lumbar spondylosis } & & \multicolumn{2}{|c|}{ Chronic pharyngitis } & \multirow{2}{*}{$\mathrm{t}$} & \multicolumn{2}{|c|}{ Neurasthenia } & \multirow{2}{*}{$\mathrm{t}$} \\
\hline & Yes & No & & Yes & No & & Yes & No & \\
\hline $\begin{array}{l}\text { Organizational } \\
\text { environment }^{*}\end{array}$ & $92.43 \pm 21.68$ & $89.15 \pm 22.32$ & $3.262^{\star *}$ & $91.96 \pm 21.80$ & $88.91 \pm 22.37$ & $2.598^{\star \star}$ & $91.82 \pm 22.03$ & $89.39 \pm 21.54$ & $2.005^{\star}$ \\
\hline Work environment & $12.47 \pm 4.45$ & $11.63 \pm 4.20$ & $4.302^{\star *}$ & $12.37 \pm 4.357$ & $11.50 \pm 4.42$ & $3.698^{* *}$ & $12.26 \pm 4.44$ & $11.90 \pm 4.16$ & 1.459 \\
\hline Facilities & $16.31 \pm 3.24$ & $15.98 \pm 3.16$ & $2.266^{*}$ & $16.24 \pm 3.18$ & $16.04 \pm 3.36$ & 1.146 & $16.26 \pm 3.24$ & $15.95 \pm 3.14$ & 1.716 \\
\hline
\end{tabular}

${ }^{*} \mathrm{P}<0.05 ;{ }^{*} \mathrm{P}<0.01 ;{ }^{*}$ Represents the total score of organizational environment questionnaire.

Table 9. Comparison of reported scores of SCL-90 with Chinese norms $(n=2111, x \pm S)$.

\begin{tabular}{|c|c|c|c|c|}
\hline Item & Medical personnel & Chinese norms & $\mathrm{t}$ & $\mathrm{p}$ \\
\hline SCL-90 & $172.53 \pm 56.36$ & $129.96 \pm 38.76$ & 34.724 & $<0.001$ \\
\hline \multicolumn{5}{|l|}{ Total score } \\
\hline SCL-90 & $1.92 \pm 0.63$ & $1.44 \pm 0.43$ & 35.018 & $<0.001$ \\
\hline \multicolumn{5}{|l|}{ Mean score } \\
\hline Positive items & $55.34 \pm 26.9$ & $24.92 \pm 18.41$ & 51.975 & $<0.001$ \\
\hline Negative items & $34.66 \pm 26.9$ & $65.08 \pm 18.33$ & -51.975 & $<0.001$ \\
\hline Mean of positive symptoms & $2.38 \pm 0.52$ & $2.60 \pm 0.59$ & -19.729 & $<0.001$ \\
\hline Somatization & $1.97 \pm 0.68$ & $1.37 \pm 0.48$ & 40.395 & $<0.001$ \\
\hline Obsession & $2.16 \pm 0.73$ & $1.62 \pm 0.58$ & 34.104 & $<0.001$ \\
\hline Interpersonal relationship & $1.92 \pm 0.68$ & $1.65 \pm 0.61$ & 18.063 & $<0.001$ \\
\hline Depression & $1.99 \pm 0.72$ & $1.50 \pm 0.59$ & 30.818 & $<0.001$ \\
\hline Anxiety & $1.82 \pm 0.66$ & $1.39 \pm 0.43$ & 29.723 & $<0.001$ \\
\hline Hostility & $1.87 \pm 0.73$ & $1.46 \pm 0.55$ & 25.718 & $<0.001$ \\
\hline Terror & $1.68 \pm 0.63$ & $1.23 \pm 0.41$ & 32.731 & $<0.001$ \\
\hline Paranoia & $1.79 \pm 0.66$ & $1.43 \pm 0.57$ & 25.580 & $<0.001$ \\
\hline Psychiatric disease & $1.81 \pm 0.61$ & $1.29 \pm 0.42$ & 39.406 & $<0.001$ \\
\hline Other & $1.93 \pm 0.66$ & I & l & l \\
\hline
\end{tabular}


Table 10. Correlation between mental health and organization environment

\begin{tabular}{|c|c|c|c|c|c|c|}
\hline Variant & $\begin{array}{c}\text { Organizational } \\
\text { environment }\end{array}$ & $\begin{array}{c}\text { Manpower } \\
\text { resource }\end{array}$ & Work environment & Facilities & $\begin{array}{c}\text { Organizational } \\
\text { culture }\end{array}$ & Management \\
\hline Somatization & $-0.356^{*}$ & $-0.386^{*}$ & $-0.369^{*}$ & $-0.308^{\star}$ & $-0.255^{\star}$ & $-0.324^{*}$ \\
\hline Obsession & $-0.339^{*}$ & $-0.382^{*}$ & $-0.361^{*}$ & $-0.288^{\star}$ & $-0.234^{*}$ & $-0.314^{*}$ \\
\hline $\begin{array}{l}\text { Interpersonal } \\
\text { relationship }\end{array}$ & $-0.340^{*}$ & $-0.351^{*}$ & $-0.345^{\star}$ & $-0.291^{\star}$ & $-0.261^{*}$ & $-0.307^{\star}$ \\
\hline Depression & $-0.378^{\star}$ & $-0.401^{*}$ & $-0.388^{\star}$ & $-0.304^{*}$ & $-0.280^{*}$ & $-0.352^{*}$ \\
\hline Anxiety & $-0.333^{*}$ & $-0.369^{*}$ & $-0.335^{\star}$ & $-0.286^{\star}$ & $-0.245^{\star}$ & $-0.301^{*}$ \\
\hline Hostility & $-0.342^{\star}$ & $-0.371^{*}$ & $-0.347^{\star}$ & $-0.287^{\star}$ & $-0.248^{\star}$ & $-0.309^{*}$ \\
\hline Terror & $-0.261^{*}$ & $-0.287^{\star}$ & $-0.238^{\star}$ & $-0.255^{\star}$ & $-0.215^{*}$ & $-0.235^{\star}$ \\
\hline Paranoia & $-0.327^{\star}$ & $-0.347^{\star}$ & $-0.325^{\star}$ & $-0.264^{*}$ & $-0.259^{*}$ & $-0.292^{*}$ \\
\hline Psychiatric disease & $-0.332^{*}$ & $-0.350^{*}$ & $-0.338^{*}$ & $-0.286^{\star}$ & $-0.264^{*}$ & $-0.300^{*}$ \\
\hline Other & $-0.344^{*}$ & $-0.368^{\star}$ & $-0.355^{*}$ & $-0.290^{*}$ & $-0.263^{*}$ & $-0.315^{\star}$ \\
\hline
\end{tabular}

Table 11. Comparison of scores of SCL-90 between different occupational injury occupational injuries.

\begin{tabular}{|c|c|c|c|c|c|c|}
\hline \multirow{2}{*}{ Variant } & \multicolumn{2}{|c|}{ Oral ulcer } & \multirow{2}{*}{$\mathrm{t}$} & \multicolumn{2}{|c|}{ Stick injury } & \multirow{2}{*}{$\mathrm{t}$} \\
\hline & Yes & No & & Yes & No & \\
\hline SCL-90 & $1.98 \pm 0.62$ & $1.81 \pm 0.62$ & $6.084^{* *}$ & $2.00 \pm 0.64$ & $1.85 \pm 0.61$ & $5.183^{\star *}$ \\
\hline Somatization & $2.03 \pm 0.69$ & $1.86 \pm 0.66$ & $5.721^{* *}$ & $2.04 \pm 0.68$ & $1.92 \pm 0.68$ & $4.043^{* *}$ \\
\hline Obsession & $2.24 \pm 0.72$ & $2.04 \pm 0.72$ & $6.189^{* *}$ & $2.23 \pm 0.73$ & $2.10 \pm 0.72$ & $4.055^{\star *}$ \\
\hline Interpersonal relationship & $1.98 \pm 0.68$ & $1.82 \pm 0.68$ & $5.472^{* *}$ & $2.00 \pm 0.69$ & $1.85 \pm 0.67$ & $4.846^{\star *}$ \\
\hline Depression & $2.06 \pm 0.73$ & $1.86 \pm 0.70$ & $6.082^{* *}$ & $2.07 \pm 0.74$ & $1.92 \pm 0.70$ & $4.992^{* *}$ \\
\hline Anxiety & $1.87 \pm 0.67$ & $1.72 \pm 0.63$ & $5.247^{\star *}$ & $1.89 \pm 0.67$ & $1.76 \pm 0.64$ & $4.753^{\star *}$ \\
\hline Hostility & $1.93 \pm 0.75$ & $1.77 \pm 0.70$ & $4.773^{* *}$ & $1.95 \pm 0.76$ & $1.81 \pm 0.71$ & $4.639^{\star *}$ \\
\hline Terror & $1.72 \pm 0.63$ & $1.60 \pm 0.61$ & $4.411^{\star *}$ & $1.74 \pm 0.65$ & $1.62 \pm 0.6$ & $4.369^{* *}$ \\
\hline Paranoia & $1.85 \pm 0.66$ & $1.70 \pm 0.64$ & $5.257^{\star *}$ & $1.88 \pm 0.68$ & $1.73 \pm 0.63$ & $5.263^{\star *}$ \\
\hline Psychiatric disease & $1.87 \pm 0.60$ & $1.72 \pm 0.61$ & $5.309^{* *}$ & $1.89 \pm 0.62$ & $1.75 \pm 0.59$ & $5.285^{\star *}$ \\
\hline Other & $2.00 \pm 0.66$ & $1.82 \pm 0.64$ & $6.068^{* *}$ & $2.02 \pm 0.68$ & $1.86 \pm 0.63$ & $5.402^{\star *}$ \\
\hline
\end{tabular}

${ }^{*} \mathrm{P}<0.01$.

Recent evidence has shown that medical error is a systemic problem requiring systemic solutions, such as the improvement of organizational environment. Therefore, developing a safety culture or safety atmosphere is one potential strategy toward improving patient safety.

In current study, we constructed a new version of organizational environment questionnaire according to the published literature and current extant research consents. To further testify the applicability of our new questionnaire, we designed and performed a large-scale survey in Zhuhai city, China, and totally 16637 medical workers were randomly sampled in 54 hospitals. Totally 2111 questionnaires were analyzed and we actually found higher rates of occupational injuries and occupational diseases in medical workers. Besides, apart from the physical health, the mental health status examined by SCL-90 also illustrated severe morbidities of behavioral diseases, including depression, anxiety, obsession, paranoia and so on. By using Pearson correlation analysis, we demonstrated that the scores of organizational environment questionnaire significantly correlated with the incidence of occupational injuries and occupational diseases, and also with the scores of SCL-90, which have also been revealed in other studies (Fan et al., 2016; Nowakowska et al., 2016; Dirik \& Intepeler, 2017). All of these data suggest that the new organizational environment questionnaire can be a reliable tool to evaluate the influence of organizational environment on medical safety in medical workers.

Although we demonstrated the efficacy of revised organizational environment questionnaire for the assessment of medical safety climate in medical workers, we still lack the particular contribution of the specific aspects of the dimensions on the occurrence of occupational injuries and occupational diseases. In the future study, we may need to evaluate the mechanisms responsible for the occurrence of occupational diseases or occupational injuries caused by dysregulation of organizational environment management. Only under this condition, can we develop and design corresponding measures to tackle with the problems of occupational safety climate on the perspective of organizational environment.

\section{Conclusions}

In this study, we revised the new version of organizational environment questionnaire and confirm its reliability and validity in a large-scale of observational investigation. We recommend the new revised organizational environment questionnaire be used in the assessment of influence of organizational environment on medical workers in China. 


\section{Acknowledgements}

This work was supported by grants from Shenzhen's Sanming Project Fund [SZSM201612018].

\section{References}

Centers for Disease Control and Prevention - CDC. (2007). Workbook for designing, implementing and evaluating a sharps injury prevention program. Retrieved from www.cdc.gov/sharpssafety/ pdf/sharpsworkbook_2008.pdf

Dirik, H. F., \& Intepeler, S. S. (2017). The work environment and empowerment as predictors of patient safety culture in Turkey. Journal of Nursing Management, 25(4), 256-265. http://dx.doi. org/10.1111/jonm.12458. PMid:28370650.

Ein Ali Afjeh, M., Pourahmad, R., Akbari-Adergani, B., \& Azin, M. (2020). Characteristics of glucose oxidase immobilized on Magnetic Chitosan Nanoparticles. Food Science and Technology, 40(1), 68-75. http://dx.doi.org/10.1590/fst.32618.

Fan, Y., Zheng, Q., Liu, S., \& Li, Q. (2016). Construction of a new model of job engagement, psychological empowerment and perceived work environment among Chinese registered nurses at four large university hospitals: implications for nurse managers seeking to enhance nursing retention and quality of care. Journal of Nursing Management, 24(5), 646-655. http://dx.doi.org/10.1111/jonm.12369. PMid:27039839.

Gershon, R. R., Stone, P. W., Zeltser, M., Faucett, J., MacDavitt, K., \& Chou, S. S. (2007). Organizational climate and nurse health outcomes in the United States: a systematic review. Industrial Health, 45(5), 622-636. http://dx.doi.org/10.2486/indhealth.45.622. PMid:18057805.

Gimeno, D., Felknor, S., Burau, K. D., \& Delclos, G. L. (2005). Organisational and occupational risk factors associated with work related injuries among public hospital employees in Costa Rica. Occupational and Environmental Medicine, 62(5), 337-343. http:// dx.doi.org/10.1136/oem.2004.014936. PMid:15837856.

Hossen, M. T., Ferdaus, M. J., Hasan, M. M., Lina, N. N., Das, A. K., Barman, S. K., Paul, D. K., \& Roy, R. K. (2021). Food safety knowledge, attitudes and practices of street food vendors in Jashore region, Bangladesh. Food Sci. Technol, 41(Suppl. 1), 226-239. http:// dx.doi.org/10.1590/fst.13320.
Nowakowska, I., Rasinska, R., \& Glowacka, M. D. (2016). The influence of factors of work environment and burnout syndrome on selfefficacy of medical staff. Annals of Agricultural and Environmental Medicine, 23(2), 304-309. http://dx.doi.org/10.5604/12321966.1203895. PMid:27294637.

Ruitenburg, M. M., Frings-Dresen, M. H., \& Sluiter, J. K. (2012). The prevalence of common mental disorders among hospital physicians and their association with self-reported work ability: a cross-sectional study. BMC Health Services Research, 12(1), 292-298. http://dx.doi. org/10.1186/1472-6963-12-292. PMid:22938170.

Schwenk, T. L., Gorenflo, D. W., \& Leja, L. M. (2008). A survey on the impact of being depressed on the professional status and mental health care of physicians. The Journal of Clinical Psychiatry, 69(4), 617-620. http://dx.doi.org/10.4088/JCP.v69n0414. PMid:18426258.

Stone, P. W., Du, Y., \& Gershon, R. R. (2007). Organizational climate and occupational health outcomes in hospital nurses. Journal of Occupational and Environmental Medicine, 49(1), 50-58. http:// dx.doi.org/10.1097/01.jom.0000251622.05429.0c. PMid:17215713.

Trinkoff, A. M., Le, R., Geiger-Brown, J., \& Lipscomb, J. (2007). Work schedule, needle use, and needlestick injuries among registered nurses. Infection Control and Hospital Epidemiology, 28(2), 156-164. http://dx.doi.org/10.1086/510785. PMid:17265396.

Trinkoff, A. M., Le, R., Geiger-Brown, J., Lipscomb, J., \& Lang, G. (2006). Longitudinal relationship of work hours, mandatory overtime, and on-call to musculoskeletal problems in nurses. American Journal of Industrial Medicine, 49(11), 964-971. http://dx.doi.org/10.1002/ ajim.20330. PMid:16691609.

Vogus, T. J., \& Sutcliffe, K. M. (2007). The Safety Organizing Scale: development and validation of a behavioral measure of safety culture in hospital nursing units. Medical Care, 45(1), 46-54. http://dx.doi. org/10.1097/01.mlr.0000244635.61178.7a. PMid:17279020.

Yildiz, E., Guldas, M., Ellergezen, P., Acar, A. G., \& Gurbuz, O. (2021). Obesity-associated pathways of anthocyanins. Food Science and Technology, 41(Suppl. 1), 1-13. http://dx.doi.org/10.1590/fst.39119.

Yoon, S. L., \& Kim, J. H. (2013). Job-related stress, emotional labor, and depressive symptoms among Korean nurses. Journal of Nursing Scholarship, 45(2), 169-176. http://dx.doi.org/10.1111/jnu.12018. PMid:23470274.

Zohar, D. (1980). Safety climate in industrial organizations: theoretical and applied implications. The Journal of Applied Psychology, 65(1), 96-102. http://dx.doi.org/10.1037/0021-9010.65.1.96. PMid:7364709. 


\section{Supplementary Material}

Supplementary material accompanies this paper.

Supplemental Table 1. The Cronbach's $\alpha$ of dimensions of organization environment questionnaire

Supplemental Table 2. The CITC and reliability analysis of dimensions of manpower resource

Supplemental Table 3. The CITC and reliability analysis of dimensions of work environment

Supplemental Table 4. The CITC and reliability analysis of dimensions of facilities

Supplemental Table 5. The CITC and reliability analysis of dimensions of organizational culture

Supplemental Table 6. The CITC and reliability analysis of dimensions of management support

Supplemental Table 7. The contributing rate of cumulative variances of 5 common factors (\%)

Supplemental Table 8. The ranking score questionnaire of dimensions of organization environment questionnaire

This material is available as part of the online article from http://www.scielo.br/cta 